\title{
SINKS AND BOUNDARY TEMPERATURES THAT NEUTRALIZE A HEAT SOURCE*
}

BY

W. A. DAY

Hertford College, Oxford

Introduction. This article is concerned with the classical theory of heat conduction.

It is envisaged that a conducting body contains a continuously distributed heat source, of density $f^{+}(x, t)$, which may vary with position $x$ in the body and with the time $t$. It is supposed, though, that only limited quantitative information about $f^{+}$is available, such as the value of the integral $\int f^{+} d x$, which is the net rate at which the source supplies heat. It is desired to neutralize the effect of the source by cooling the body and arranging that the temperature $u(x, t)$ be negative at each point $x$, at least for all sufficiently large $t$. Two alternative strategies for cooling the body will be considered: the first is to install a continuously distributed heat sink, of density $f^{-}(x, t)$ and maintain the boundary at zero temperature; the second is to control the temperature $\tau(t)$ of the boundary itself.

It will turn out that there is an essential difference between the information required about $f^{+}(x, t)$ when $x$ is a point of $\mathbf{R}^{1}$ and when it is a point of $\mathbf{R}^{n}$, with $n>1$.

\section{Temperatures on an interval}

1. Sinks that neutralize a source. The simplest case $(n=1)$ is that of conduction in an isotropic, but possibly inhomogeneous, slab, which may be identified with an interval, $a \leqslant x \leqslant b$, say. The temperature $u(x, t)(a \leqslant x \leqslant b, 0<t<\infty)$ is required to satisfy the differential equation

$$
\frac{\partial}{\partial x}\left(k(x) \frac{\partial u}{\partial x}\right)=c(x) \frac{\partial u}{\partial t}+f^{-}(x, t)-f^{+}(x, t),
$$

and the boundary condition

$$
u(a, t)=u(b, t)=0 \quad(t>0),
$$

\footnotetext{
${ }^{*}$ Received January 28, 1986.
} 
and it is supposed that the thermal conductivity $k$ and the specific heat $c$ are positive, while $f^{+}$and $f^{-}$are nonnegative and continuously differentiable.

No initial condition has been imposed upon the temperature and, consequently, there will be infinitely many temperatures $u$ which correspond to a given source $f^{+}$and a given sink $f^{-}$.

In view of the boundary condition (1.2), the maximum temperature cannot be negative, that is to say,

$$
(\max u)(t)=\max \{u(x, t): a \leqslant x \leqslant b\} \geqslant 0 .
$$

We seek to choose $f^{-}$in such a way that the maximum temperature decays to zero and, in fact, we shall say that $f^{-}$neutralizes $f^{+}$if every $u$ that satisfies (1.1) and (1.2) has the property that $\max u \rightarrow 0$ as $t \rightarrow \infty$.

Related considerations arise in the static theory of elastic beams, where it is desired to shore up a beam against an applied load but details of the loading are not available and only the net load is known. For a discussion of these matters the reader is referred to my article [1], and to S. Friedland's article [2] printed immediately after my own.

There is a very simple condition which ensures that $f^{-}$neutralizes $f^{+}$.

THEOREM 1. The condition $f^{-} \geqslant f^{+}$is sufficient to ensure that $f^{-}$neutralizes $f^{+}$and, in particular, the condition $f^{-}=f^{+}$is sufficient.

Theorem 1 provides a straightforward strategy for cooling the slab, but we regard it as unsatisfactory for the reason that such a choice as $f^{-}=f^{+}$demands the maximum possible information about $f^{+}$, namely, the value of $f^{+}(x, t)$ at each $x$ in $a \leqslant x \leqslant b$ and at each $t>0$. Nonetheless the theorem plays an important role in devising more satisfactory strategies, and we shall record a proof of it.

Let $u$ satisfy (1.1) and (1.2), let $t_{0}>0$ be arbitrary, let $v$ be the solution of the boundary- and initial-value problem

$$
\begin{aligned}
& \frac{\partial}{\partial x}\left(k \frac{\partial v}{\partial x}\right)=c \frac{\partial v}{\partial t} \\
& v(a, t)=v(b, t)=0 \quad\left(t>t_{0}\right), \\
& v\left(x, t_{0}\right)=u\left(x, t_{0}\right) \quad(a \leqslant x \leqslant b) \text {, }
\end{aligned}
$$

in which the differential equation and the boundary conditions are homogeneous but the initial condition is not, and let $w=u-v$. As is well known, $\max |v| \rightarrow 0$ as $t \rightarrow \infty$. On substituting $v+w$ for $u$ in (1.1) we see that $w$ is a solution of the equation

$$
\frac{\partial}{\partial x}\left(k \frac{\partial w}{\partial x}\right)=c \frac{\partial w}{\partial t}+f^{-}-f^{+}
$$

and, therefore, satisfies the inequality

$$
\frac{\partial}{\partial x}\left(k \frac{\partial w}{\partial x}\right) \geqslant c \frac{\partial w}{\partial t}
$$


if $f^{-} \geqslant f^{+}$. Since $w$ also satisfies the homogeneous boundary and initial conditions

$$
\begin{aligned}
& w(a, t)=w(b, t)=0 \quad\left(t>t_{0}\right), \\
& w\left(x, t_{0}\right)=0 \quad(a \leqslant x \leqslant b),
\end{aligned}
$$

the Maximum Principle implies that $w \leqslant 0$ throughout the half-strip $a \leqslant x \leqslant b, t_{0} \leqslant t<$ $\infty$. Thus, $u=v+w \leqslant v \leqslant \max |v|$ in the half-strip and, therefore, $0 \leqslant \max u \leqslant \max |v|$. It follows that $\max u \rightarrow 0$ as $t \rightarrow \infty$ and, hence, it is the case that $f^{-}$neutralizes $f^{+}$.

In order to produce a more satisfactory strategy for choosing $f^{-}$it is convenient to introduce the functions

$$
\phi(x)=\frac{1}{\gamma} \int_{x}^{b} \frac{d \xi}{k(\xi)}, \quad \psi(x)=\frac{1}{\gamma} \int_{a}^{x} \frac{d \xi}{k(\xi)},
$$

where $\gamma$ is the total thermal resistivity

$$
\int_{a}^{b} \frac{d \xi}{k(\xi)}
$$

and a kernel $G(x, y)$ which equals

$$
\begin{array}{ll}
\gamma \phi(x) \psi(y) & (a \leqslant y \leqslant x \leqslant b), \\
\gamma \psi(x) \phi(y) & (a \leqslant x \leqslant y \leqslant b) .
\end{array}
$$

The kernel is the Green's function associated with the boundary value problem

$$
\frac{d}{d x}\left(k \frac{d g}{d x}\right)=-f, \quad g(a)=g(b)=0,
$$

the solution being

$$
g(x)=\int_{a}^{b} G(x, y) f(y) d y .
$$

Theorems 2 and 3 are concerned with what happens when $f^{+}$is known to be a monotone function of $t$ (in the wider sense), for each fixed $x$. In the first case $f^{+}$increases

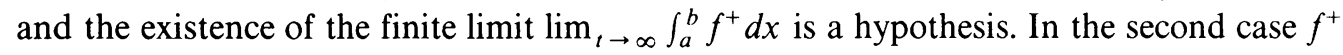
decreases and the existence of the limit is guaranteed because $f^{+} \geqslant 0$.

TheOREM 2. Suppose that $f^{+}(\geqslant 0)$ increases as $t$ increases, and $\lim _{t \rightarrow \infty} \int_{a}^{b} f^{+} d x$ exists. Then, in order that $f^{-}$should neutralize $f^{+}$it is sufficient that

(i) $f^{-}(x)(\geqslant 0)$ be independent of $t$, and

(ii) $\int_{a}^{b} \phi(x) \psi(x) f^{-}(x) d x=\lim _{t \rightarrow \infty} \int_{a}^{b} f^{+}(x, t) d x$.

Some remarks are in order before we proceed to the proof of the theorem.

The presence, in the integral involving $f^{-}$, of some such weight as $\phi(x) \psi(x)$, which vanishes at $x=a$ and $x=b$ but is positive in $a<x<b$, is to be expected; it would be wasteful to concentrate $f^{-}$too near a face of the slab.

Next, it will be noticed that the only quantitative information demanded of $f^{+}$is the value of $\lim _{t \rightarrow \infty} \int_{a}^{b} f^{+} d x$, which is the ultimate net rate at which the source supplies heat.

Finally, if we were to choose $f^{-}=f^{+}$, as Theorem 1 permits, the net rate at which the sink extracts heat would balance the net rate at which the source supplies heat, that is to say, it would be the case that

$$
\int_{a}^{b} f^{-} d x=\int_{a}^{b} f^{+} d x
$$


However, the definitions of $\phi$ and $\psi$ imply that $\phi+\psi=1$ and, hence, that $4 \phi \psi \leqslant(\phi+$ $\psi)^{2}=1$. This bound on the weight has the consequence that if $f^{-}$be chosen in accordance with the rules laid down in Theorem 2 then

$$
\int_{a}^{b} f^{-} d x \geqslant 4 \lim _{t \rightarrow \infty} \int_{a}^{b} f^{+} d x .
$$

The large factor 4 is the penalty for attempting to make do with little information about $f^{+}$.

The proof of Theorem 2 hinges upon the estimates

$$
\gamma \phi(x) \psi(x) \phi(y) \psi(y) \leqslant G(x, y) \leqslant \gamma \phi(x) \psi(x)
$$

on the kernel. To verify these, consider the behavior of the kernel in the triangle $a \leqslant y \leqslant x \leqslant b$. Since $\psi$ increases, it must be that

$$
G(x, y)=\gamma \phi(x) \psi(y) \leqslant \gamma \phi(x) \psi(x),
$$

which is the upper bound. On the other hand, a glance at the definitions of $\phi$ and $\psi$ assures us that $0 \leqslant \phi(y) \leqslant 1$ and $0 \leqslant \psi(x) \leqslant 1$. Therefore,

$$
G(x, y)=\gamma \phi(x) \psi(y) \geqslant \gamma \phi(x) \psi(x) \phi(y) \psi(y),
$$

which is the lower bound. Thus, the estimates hold in $a \leqslant y \leqslant x \leqslant b$, and a similar argument establishes that they also hold in the complementary triangle $a \leqslant x \leqslant y \leqslant b$.

Now let $u$ be any solution of (1.1) that satisfies the boundary conditions (1.2), and introduce

$$
v(x, t)=\int_{a}^{b} G(x, y)\left(f^{+}(y, t)-f^{-}(y)\right) d y
$$

and the difference $w=u-v$. Since $f^{+}$and $f^{-}$are nonnegative the estimates (1.3) imply that

$$
v(x, t) \leqslant \gamma \phi(x) \psi(x)\left[\int_{a}^{b} f^{+}(y, t) d y-\int_{a}^{b} \phi(y) \psi(y) f^{-}(y) d y\right],
$$

and the hypotheses of the theorem ensure that the quantity enclosed within square brackets is not positive. Thus $v \leqslant 0, u=v+w \leqslant w$, and $0 \leqslant \max u \leqslant \max w$.

On differentiating the formula that defines $v$ we obtain

$$
\frac{\partial v}{\partial t}(x, t)=\int_{a}^{b} G(x, y) \frac{\partial f^{+}}{\partial t}(y, t) d y \geqslant 0 .
$$

Accordingly, if we substitute $v+w$ for $u$ in (1.1) and use this last inequality we deduce that

$$
\begin{aligned}
\frac{\partial}{\partial x}\left(k \frac{\partial v}{\partial x}\right)+\frac{\partial}{\partial x}\left(k \frac{\partial w}{\partial x}\right) & =c \frac{\partial v}{\partial t}+c \frac{\partial w}{\partial t}+f^{-}-f^{+} \\
& \geqslant c \frac{\partial w}{\partial t}+f^{-}-f^{+} .
\end{aligned}
$$

However, the definition of $v$ implies the equation

$$
\frac{\partial}{\partial x}\left(k \frac{\partial v}{\partial x}\right)=f^{-}-f^{+}
$$


and, thus, $w$ satisfies the inequality

$$
\frac{\partial}{\partial x}\left(k \frac{\partial w}{\partial x}\right) \geqslant c \frac{\partial w}{\partial t}
$$

The definition of $v$ also implies that $v(a, t)=v(b, t)=0(t>0)$, and, in view of (1.2). it must be that $w(a, t)=w(b, t)=0(t>0)$. By virtue of Theorem 1, max $w \rightarrow 0$ as $t \rightarrow \infty$. and since, as we have seen, $0 \leqslant \max u \leqslant \max w$ it must be that $\max u \rightarrow 0$ as $t \rightarrow \infty$. In short, $f^{-}$neutralizes $f^{+}$.

Next we consider what happens when $f^{+}$decreases.

Theorem 3. Suppose that $f^{+}(\geqslant 0)$ decreases as $t$ increases. Then, in order that $f^{--}$should neutralize $f^{+}$it is sufficient that

(i) $f^{-}(\geqslant 0)$ should decrease as $t$ increases, and

(ii) $\int_{a}^{b} \phi(x) \psi(x) f^{-}(x, t) d x=\int_{a}^{b} f^{+}(x, t) d x(t>0)$.

The proof proceeds on similar lines to that of Theorem 2. We suppose $u$ to be a solution of (1.1) which satisfies (1.2) and put $w=u-v$, where

$$
v(x, t)=\int_{a}^{b} G(x, y)\left(f^{+}(y, t)-f^{-}(y, t)\right) d y .
$$

It follows, just as before, that

$$
\frac{\partial}{\partial x}\left(k \frac{\partial v}{\partial x}\right)=f^{-}-f^{+}, \quad v(a, t)=v(b, t)=0 \quad(t>0),
$$

and it is necessary to verify only that $v \leqslant 0$ and $\partial v / \partial t \geqslant 0$, for a repetition of our earlier argument will then enable us to conclude that $f^{-}$neutralizes $f^{+}$.

Since $f^{+}$and $f^{-}$are nonnegative the estimates (1.3) yield the inequality

$$
v(x, t) \leqslant \gamma \phi(x) \psi(x)\left[\int_{a}^{b} f^{+}(y, t) d y-\int_{a}^{b} \phi(y) \psi(y) f^{-}(y, t) d y\right]=0 .
$$

On the other hand, because $\partial f^{+} / \partial t$ and $\partial f^{-} / \partial t$ are nonpositive

$$
\begin{aligned}
\frac{\partial v}{\partial t}(x, t) & =\int_{a}^{b} G(x, y)\left(\frac{\partial f^{+}}{\partial t}(y, t)-\frac{\partial f^{-}}{\partial t}(y, t)\right) d y \\
& \geqslant \gamma \phi(x)\left[\int_{a}^{b} \frac{\partial f^{+}}{\partial t}(y, t) d y-\int_{a}^{b} \phi(y) \psi(y) \frac{\partial f^{-}}{\partial t}(y, t) d y\right] \\
& =\gamma \phi(x) \psi(x) \frac{d}{d t}\left[\int_{a}^{b} f^{+}(y, t) d y-\int_{a}^{b} \phi(y) \psi(y) f^{-}(y, t) d y\right] \\
& =0
\end{aligned}
$$

and, hence, $f^{-}$neutralizes $f^{+}$.

2. Boundary temperatures that neutralize a source. Much the same circle of ideas can be used to treat the problem of neutralizing $f^{+}$by controlling the boundary temperature. The differential equation (1.1) is now replaced by the equation

$$
\frac{\partial}{\partial x}\left(k(x) \frac{\partial u}{\partial x}\right)=c(x) \frac{\partial u}{\partial t}-f^{+}(x, t),
$$


and the boundary condition (1.2) is replaced by

$$
u(a, t)=u(b, t)=\tau(t) \quad(t>0) .
$$

We shall say that $\tau$ neutralizes $f^{+}$if every $u$ that satisfies (2.1) and (2.2) has the property that $\limsup _{t \rightarrow \infty}(\max u) \leqslant 0$.

Theorem 4 shows that, whenever $f^{+}$satisfies the same hypotheses as in Theorem 2 , it is enough to take $\tau$ to be an appropriate constant.

TheOREM 4. Suppose that $f^{+}(\geqslant 0)$ increases as $t$ increases, and $\lim _{t \rightarrow \infty} \int_{a}^{b} f^{+} d x$ exists. Then, in order that $\tau$ should neutralize $f^{+}$it is sufficient that

$$
\tau=-\frac{1}{4} \gamma \lim _{t \rightarrow \infty} \int_{a}^{b} f^{+}(x, t) d x .
$$

To prove this let $\tau$ be the constant of the theorem, let $u$ satisfy (2.1) and (2.2), let

$$
v(x, t)=\int_{a}^{b} G(x, y) f^{+}(y, t) d y,
$$

and let $w=u-\tau-v$. The definition of $v$ implies that

$$
\frac{\partial}{\partial x}\left(k \frac{\partial v}{\partial x}\right)=-f^{+}, \quad v(a, t)=v(b, t)=0 \quad(t>0),
$$

and, on substituting $\tau+v+w$ for $u$ in (2.1) and (2.2), we find that

$$
\frac{\partial}{\partial x}\left(k \frac{\partial w}{\partial x}\right)=c \frac{\partial v}{\partial t}+c \frac{\partial w}{\partial t}, \quad w(a, t)=w(b, t)=0 \quad(t>0) .
$$

Since

$$
\frac{\partial v}{\partial t}(x, t)=\int_{a}^{b} G(x, y) \frac{\partial f^{+}}{\partial t}(y, t) d y \geqslant 0
$$

it follows that

$$
\frac{\partial}{\partial x}\left(k \frac{\partial w}{\partial x}\right) \geqslant c \frac{\partial w}{\partial t}
$$

and Theorem 1 tells us that $\max w \rightarrow 0$ as $t \rightarrow \infty$.

Moreover, the upper bounds on $G$ and $\phi \psi$ that have been used already imply that

$$
v(x, t) \leqslant \gamma \phi(x) \psi(x) \int_{a}^{b} f^{+}(y, t) d y \leqslant \frac{1}{4} \gamma \int_{a}^{b} f^{+}(y, t) d y
$$

and, therefore,

$$
\max v \leqslant \frac{1}{4} \gamma \lim _{t \rightarrow \infty} \int_{a}^{b} f^{+} d x=-\tau .
$$

Because $u=\tau+v+w$ it must be that $\max u \leqslant \tau+\max v+\max w \leqslant \max w$ and, hence, $\limsup _{t \rightarrow \infty}(\max u) \leqslant 0$. In summary, $\tau$ neutralizes $f^{+}$.

The next theorem deals with what happens when $f^{+}$decreases.

TheOREM 5. Suppose that $f^{+}(\geqslant 0)$ decreases as $t$ increases. Then, in order that $\tau$ should neutralize $f^{+}$it is sufficient that

$$
\tau(t)=-\frac{1}{4} \gamma \int_{a}^{b} f^{+}(x, t) d x \quad(t>0) .
$$


The proof is much like that of Theorem 4. Indeed, let $u$ satisfy (2.1) and (2.2), and let $v$ and $w$ be defined as before. On substituting $\tau+v+w$ for $u$ in (2.1) we obtain

$$
\frac{\partial}{\partial x}\left(k \frac{\partial w}{\partial x}\right)=c \frac{d \tau}{d t}+c \frac{\partial v}{\partial t}+c \frac{\partial w}{\partial t} .
$$

However,

$$
\begin{aligned}
\frac{d \tau}{d t}(t)+\frac{\partial v}{\partial t}(x, t) & =-\frac{1}{4} \gamma \int_{a}^{b} \frac{\partial f^{+}}{\partial t}(y, t) d y+\int_{a}^{b} G(x, y) \frac{\partial f^{+}}{\partial t}(y, t) d y \\
& =\frac{1}{4} \gamma \int_{a}^{b}\left|\frac{\partial f^{+}}{\partial t}(y, t)\right| d y-\int_{a}^{b} G(x, y)\left|\frac{\partial f^{+}}{\partial t}(y, t)\right| d y \\
& \geqslant 0
\end{aligned}
$$

and, hence,

$$
\frac{\partial}{\partial x}\left(k \frac{\partial w}{\partial x}\right) \geqslant c \frac{\partial w}{\partial t} .
$$

Since, once again, $w(a, t)=w(b, t)=0(t>0)$, Theorem 1 implies that $\max w \rightarrow 0$ as $t \rightarrow \infty$. Lastly, we note that

$$
v(x, t) \leqslant \frac{1}{4} \gamma \int_{a}^{b} f^{+}(y, t) d y=-\tau(t)
$$

and, therefore, $\max v \leqslant-\tau$ and $\max u \leqslant \tau+\max v+\max w \leqslant \max w$. Hence $\lim \sup _{t \rightarrow \infty}(\max u) \leqslant 0$ and the proof is complete.

\section{Temperatures in a ball}

We turn to considering what happens in $\mathbf{R}^{n}$; our methods will enable us to deal with only the simplest case but even here a new feature is encountered. In what follows, the interval $a \leqslant x \leqslant b$ is replaced by the ball $B=\{x:|x| \leqslant a\}$, and the thermal conductivity $k$ and the specific heat $c$ are positive constants. For ease of exposition it is supposed that $n>2$; nevertheless the arguments can be carried through when $n=2$ by making the minor modifications necessitated by the fact that the singular solution of Laplace's equation is logarithmic in character.

3. Sinks that neutralize a source. This section is given over to proving a counterpart to Theorem 2. Equation (1.1) is now replaced by the equation

$$
k \Delta u=c \frac{\partial u}{\partial t}+f^{-}-f^{+}
$$

and the boundary condition

$$
u(x, t)=0 \quad(|x|=a, t>0)
$$

is supposed to be in force.

The novel feature which enters in higher dimensions is that the quantitative information required about the source is not the value of the integral

$$
\int_{B} f^{+} d x
$$


but the value of a Lebesgue norm

$$
\left(\int_{B}\left|f^{+}\right|^{p} d x\right)^{1 / p}
$$

where $p>n$ but $p$ is otherwise arbitrary. It is convenient to introduce the constant

$$
\Omega(p)=2^{n+1}\left[\frac{\omega_{n} a^{n}(p-1)^{-}}{p-n}\right]^{(p-1) / p},
$$

$\omega_{n}=2 \pi^{n / 2} / \Gamma\left(\frac{1}{2} n\right)$ being the hyperarea of the unit sphere $\{x:|x|=1\}$.

As before, we aim to ensure that $f^{-}$neutralizes $f^{+}$in the sense that every $u$ that satisfies (3.1) and (3.20) has the property that $\max u \rightarrow 0$ as $t \rightarrow \infty$.

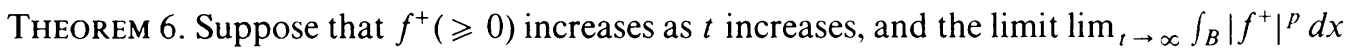
exists for some $p>n$. Then, in order that $f^{-}$should neutralize $f^{+}$it is sufficient that

(i) $f^{-}(x)(\geqslant 0)$ be independent of $t$, and

(ii) $\int_{B}(\mathrm{i}-|x| / a) f^{-}(x) d x=\Omega(p) \lim _{t \rightarrow \infty}\left(\int_{B}\left|f^{+}(x, t)\right|^{p} d x\right)^{1 / p}$.

Certain comments are required before we prove the theorem.

It will be noticed, once again, that a weight occurs in the integral involving $f^{-}$.

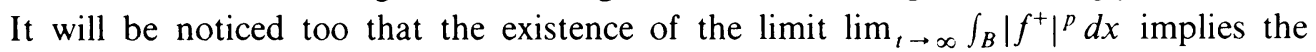
existence of $\lim _{t \rightarrow \infty} \int_{B}\left|f^{+}\right|^{p^{\prime}} d x$ for every $p^{\prime}$ in $1 \leqslant p^{\prime} \leqslant p$, but not necessarily for $p^{\prime}>p$.

Finally, Hölder's inequality tells us that

$$
\int_{B} f^{+} d x \leqslant\left(\int_{B} d x\right)^{(p-1) / p}\left(\int_{B}\left|f^{+}\right|^{p} d x\right)^{1 / p}=\left(\frac{\omega_{n} a^{n}}{n}\right)^{(p-1) / p}\left(\int_{B}\left|f^{+}\right|^{p} d x\right)^{1 / p}
$$

and, therefore, if $f^{-}$is chosen to satisfy condition (ii) its integral must satisfy

$$
\begin{aligned}
\int_{B} f^{-}(x) d x & \geqslant \int_{B}\left(1-\frac{|x|}{a}\right) f^{-}(x) d x \\
& =\Omega(p) \lim _{t \rightarrow \infty}\left(\int_{B}\left|f^{+}(x, t)\right|^{p} d x\right)^{1 / p} \\
& \geqslant \Omega(p)\left(\frac{n}{\omega_{n} a^{n}}\right)^{(p-1) / p} \lim _{t \rightarrow \infty} \int_{B} f^{+}(x, t) d x \\
& =2^{n+1}\left[\frac{n(p-1)}{p-n}\right]^{(p-1) / p} \lim _{t \rightarrow \infty} \int_{B} f^{+}(x, t) d x .
\end{aligned}
$$

Since $p>n>2$ we can use the inequality $(1+\theta)^{\eta} \geqslant 1+\theta \eta(\theta \geqslant 0, \eta \geqslant 1)$, with $\theta=(n-1) /(p-n)$ and $\eta=p-1$, to deduce that

$$
\left[\frac{p-1}{p-n}\right]^{p-1}=\left[1+\frac{n-1}{p-n}\right]^{p-1} \geqslant 1+(p-1)\left(\frac{n-1}{p-n}\right) \geqslant 1+n-1=n
$$

and, hence,

$$
\left[\frac{n(p-1)}{p-n}\right]^{(p-1) / p} \geqslant n^{(p-1) / p} \cdot n^{1 / p}=n .
$$


Thus, the prescription of the theorem entails that

$$
\int_{B} f^{-}(x) d x \geqslant n 2^{n+1} \lim _{t \rightarrow \infty} \int_{B} f^{+}(x, t) d x
$$

and the presence of the large factor $n 2^{n+1}$ reflects our ignorance of the details of $f^{+}$.

The proof of Theorem 2 hinges upon the estimates (1.3) and, in much the same way, Theorem 6 depends upon certain estimates on the kernel that occurs in the integral representation

$$
g(x)=\int_{B} G(x, y) f(y) d y
$$

of the solution of the boundary value problem

$$
\Delta g=-f, \quad g(x)=0 \quad \text { if }|x|=a,
$$

for Poisson's equation.

In order to prove corresponding results for regions whose geometry is more complicated it would be desirable to construct arguments that do not depend upon detailed knowledge of the kernel.

In this case, though, we can start from the fact that

$$
(n-2) \omega_{n} G=D^{2-n}-E^{2-n},
$$

where

$$
\begin{aligned}
& D(x, y)=|x-y| \\
& E(x, y)=\frac{1}{a}|x|\left|x^{*}-y\right|=\frac{1}{a}|y|\left|x-y^{*}\right|,
\end{aligned}
$$

$x$ and $y$ being distinct points of $B$. The asterisk denotes inversion, that is to say,

$$
x^{*}=\frac{a^{2}}{|x|^{2}} x \quad(x \neq 0) \text {. }
$$

The identity

$$
E(x, y)^{2}=D(x, y)^{2}+\frac{1}{a^{2}}\left(a^{2}-|x|^{2}\right)\left(a^{2}-|y|^{2}\right),
$$

which is easily verified, is crucial, and so are the bounds

$$
\frac{1}{2 a}\left(a^{2}-|y|^{2}\right) \leqslant E(x, y) \leqslant a+|y| \leqslant 2 a .
$$

To prove (3.4), we use the formula $E(x, y)=\frac{1}{a}|y|\left|x-y^{*}\right|$. The maximum value of $E(x, y)$ with respect to $x$ occurs when $x$ is the point of $B$ furthest from $y^{*}$, that is to say when $x=\frac{-a}{|\cdot|} y$. Thus

$$
E(x, y) \leqslant E\left(-\frac{a}{|y|} y, y\right)=a+|y| \leqslant 2 a .
$$

Likewise the minimum value of $E(x, y)$ with respect to $x$ occurs when $x$ is the point of $B$ closest to $y^{*}$, that is to say when $x=\frac{a}{|\cdot| \cdot \mid} y$, and

$$
E(x, y) \geqslant E\left(\frac{a}{|y|} y, y\right)=a-|y|=\frac{a^{2}-|y|^{2}}{a+|y|} \geqslant \frac{1}{2 a}\left(a^{2}-|y|^{2}\right) .
$$


To obtain an upper bound on $G$ we appeal to the inequality

$$
A^{n-2}-B^{n-2} \leqslant(n-2)(A-B) A^{n-3} \quad(0 \leqslant B \leqslant A),
$$

with $A=D^{-1}$ and $B=E^{-1}$, and get

$$
\begin{aligned}
(n-2) \omega_{n} G & \leqslant(n-2)\left(\frac{1}{D}-\frac{1}{E}\right) \frac{1}{D^{n-3}} \\
& =\frac{(n-2)(E-D)}{D^{n-2} E} \\
& =\frac{(n-2)\left(E^{2}-D^{2}\right)}{D^{n-2} E(E+D)} .
\end{aligned}
$$

On dividing through by $(n-2) \omega_{n}$, substituting for $E^{2}-D^{2}$ from (3.3), and replacing the factor $E+D$ in the denominator by $D$, which is smaller, we deduce that

$$
G(x, y) \leqslant \frac{\left(a^{2}-|x|^{2}\right)\left(a^{2}-|y|^{2}\right)}{\omega_{n} a^{2}|x-y|^{n-1} E(x, y)} .
$$

Lastly, we appeal to the lower bound on $E$ that (3.4) supplies and arrive at the inequality

$$
G(x, y) \leqslant \frac{2\left(a^{2}-|x|^{2}\right)}{\omega_{n} a|x-y|^{n-1}} .
$$

The derivation of a lower bound depends upon writing

$$
(n-2) \omega_{n} G=\frac{1}{E^{n-2}}\left(\frac{1}{(1-F)^{(n-2) / 2}}-1\right),
$$

where $F=\left(E^{2}-D^{2}\right) / E^{2}$, and using the inequality

$$
(1-F)^{-(n-2) / 2} \geqslant 1+\frac{1}{2}(n-2) F \quad(0 \leqslant F<1)
$$

to obtain

$$
G \geqslant\left(E^{2}-D^{2}\right) / 2 \omega_{n} E^{n},
$$

where we have divided through by $(n-2) \omega_{n}$. When we substitute for $E^{2}-D^{2}$ from (3.3), and appeal to the upper bounds on $E$, we see that

$$
G(x, y) \geqslant \frac{\left(a^{2}-|x|^{2}\right)\left(a^{2}-|y|^{2}\right)}{2 \omega_{n} a^{2}(a+|y|)^{n}}=\frac{\left(a^{2}-|x|^{2}\right)(a-|y|)}{2 \omega_{n} a^{2}(a+|y|)^{n-1}},
$$

and in this way we arrive at the inequality

$$
G(x, y) \geqslant \frac{\left(a^{2}-|x|^{2}\right)(a-|y|)}{2^{n} \omega_{n} a^{n+1}} .
$$

To complete the proof of Theorem 6, let $u$ satisfy the differential equation (3.1) and the boundary condition (3.2), and let $w=u-v$, where

$$
k v(x, t)=\int_{B} G(x, y)\left(f^{+}(y, t)-f^{-}(y)\right) d y .
$$


We shall show that $v \leqslant 0$. Since $f^{+}$and $f^{-}$are nonnegative, the estimates (3.5) and (3.6) imply that

$$
k v(x, t) \leqslant \frac{\left(a^{2}-|x|^{2}\right)}{2^{n} \omega_{n} a^{n}}\left[2^{n+1} a^{n-1} \int_{B} \frac{f^{+}(y, t)}{|-y|^{n-1}} d y-\int_{B}\left(1-\frac{|y|}{a}\right) f^{-}(y) d y\right] .
$$

If now $p>n$ and $p^{-1}+q^{-1}=1$, Hölder's inequality yields

$$
\int_{B} \frac{f^{+}(y, t)}{|x-y|^{n-1}} d y \leqslant\left(\int_{B}\left|f^{+}(y, t)\right|^{p} d y\right)^{1 / p}\left(\int_{B} \frac{d y}{|x-y|^{q(n-1)}}\right)^{1 / q} .
$$

However, $q(n-1)<n$ if $p>n$ and, by virtue of a known estimate on improper integrals (Hellwig [3], p.172),

$$
\begin{aligned}
\int_{B} \frac{d y}{|x-y|^{q(n-1)}} & \leqslant \int_{B} \frac{d y}{|y|^{q(n-1)}} \\
& =\int_{0}^{a} \frac{\omega_{n} r^{n-1}}{r^{q(n-1)}} d r \\
& =\frac{\omega_{n} a^{n-q(n-1)}}{n-q(n-1)}=\left(\frac{p-1}{p-n}\right) \omega_{n} a^{(p-n) /(p-1)} .
\end{aligned}
$$

Thus $k v(x, t)$ does not exceed

$$
\begin{array}{r}
\frac{\left(a^{2}-|x|^{2}\right)}{2^{n} \omega_{n} a^{n}}\left[2^{n+1} a^{n-1}\left(\frac{\omega_{n}(p-1)}{p-n}\right)^{(p-1) / p} a^{(p-n) / p}\left(\int_{B}\left|f^{+}(y, t)\right|^{p} d y\right)^{1 / p}\right. \\
\left.\quad-\int_{B}\left(1-\frac{|y|}{a} f^{-}(y)\right) d y\right] \\
=\frac{\left(a^{2}-|x|^{2}\right)}{2^{n} \omega_{n} a^{n}}\left[\Omega(p)\left(\int_{B}\left|f^{+}(y, t)\right|^{p} d y\right)^{1 / p}-\int_{B}\left(1-\frac{|y|}{a}\right) f^{-}(y) d y\right]
\end{array}
$$

and it follows from hypothesis (ii) that $v \leqslant 0$. Therefore, $u=v+w \leqslant w$ and $0 \leqslant \max u$ $\leqslant \max w$.

It is also the case that $\partial v / \partial t \geqslant 0$, because

$$
k \frac{\partial v}{\partial t}(x, t)=\int_{B} G(x, y) \frac{\partial f^{+}}{\partial t}(y, t) d y,
$$

and $f^{+}$increases as $t$ increases. Moreover, the definition of $v$ ensures that

$$
k \Delta v=f^{-}-f^{+}, \quad v(x, t)=0 \quad(|x|=a, t>0),
$$

and, on substituting $v+w$ for $u$ in (3.1) and (3.2), we deduce that

$$
k \Delta w \geqslant c \frac{\partial w}{\partial t}, \quad w(x, t)=0 \quad(|x|=a, t>0),
$$

and, therefore $\max w \rightarrow 0$ as $t \rightarrow \infty$. Thus $\max u \rightarrow 0$, and we have confirmed that $f^{-}$ neutralizes $f^{+}$. 
4. Boundary temperatures that neutralize a source. In this case the differential equation is

$$
k \Delta u=c \frac{\partial u}{\partial t}-f^{+}
$$

the boundary condition is

$$
u(x, t)=\tau(t) \quad(|x|=a, t>0),
$$

and $\tau$ will be said to neutralize $f^{+}$if every $u$ that satisfies (4.1) and (4.2) has the property that $\lim \sup _{t \rightarrow \infty}(\max u) \leqslant 0$.

If the source satisfies the same hypothesis as in Theorem 6 it is enough to take $\tau$ to be a suitable constant.

THEOREM 7. Suppose that $f^{+}(\geqslant 0)$ increases as $t$ increases, and the $\operatorname{limit}_{t \rightarrow \infty} \int_{B}\left|f^{+}\right|^{p} d x$ exists for some $p>n$. Then in order that $\tau$ should neutralize $f^{+}$it is sufficient that

$$
\tau=-\frac{\Omega(p)}{2^{n} k \omega_{n} a^{n-2}} \lim _{t \rightarrow \infty}\left(\int_{B}\left|f^{+}(x, t)\right|^{p} d x\right)^{1 / p} .
$$

Let $\tau$ be the constant just defined, let $u$ satisfy (4.1) and (4.2), let

$$
k v(x, t)=\int_{B} G(x, y) f^{+}(y, t) d y,
$$

and let $w=u-\tau-v$. By making only trivial changes to the proof of Theorem 4, we deduce that

$$
k \Delta w \geqslant c \frac{\partial w}{\partial t}, \quad w(x, t)=0 \quad(|x|=a, t>0),
$$

and, hence, that $\max w \rightarrow 0$ as $t \rightarrow \infty$.

The upper bound (3.5) on the kernel, and the arguments used in proving Theorem 6, enable us to conclude that $k v(x, t)$ does not exceed

$$
\begin{aligned}
& \frac{2\left(a^{2}-|x|^{2}\right)}{\omega_{n} a} \int_{B} \frac{f^{+}(y, t)}{|x-y|^{n-1}} d y \\
& \leqslant \frac{2 a}{\omega_{n}} \int_{B} \frac{f^{+}(y, t)}{|x-y|^{n-1}} d y \\
& \leqslant \frac{2 a}{\omega_{n}}\left(\left(\frac{p-1}{p-n}\right) \omega_{n} a^{(p-n) /(p-1)}\right)^{(p-1) / p}\left(\int_{B}\left|f^{+}(y, t)\right|^{p} d y\right)^{1 / p} \\
& =\frac{\Omega(p)}{2^{n} \omega_{n} a^{n-2}}\left(\int_{B}\left|f^{+}(y, t)\right|^{p} d y\right)^{1 / p} \leqslant-k \tau .
\end{aligned}
$$

Thus $\max v \leqslant-\tau$ and, because $u=\tau+v+w$, it must be that $\max u \leqslant \tau+\max v+$ $\max w \leqslant \max w$. It follows that $\lim \sup _{t \rightarrow \infty}(\max u) \leqslant 0$; in other words, $\tau$ neutralizes $f^{+}$ and the proof is complete. 
If we use Hölder's inequality, and argue as we did in commenting on Theorem 6, we see that

$$
\begin{aligned}
& \frac{\Omega(p)}{2^{n} \omega_{n} a^{n-2}}\left(\int_{B}\left|f^{+}(x, t)\right|^{p} d x\right)^{1 / p} \\
& \geqslant \frac{\Omega(p)}{2^{n} \omega_{n} a^{n-2}} \cdot\left(\frac{n}{\omega_{n} a^{n}}\right)^{(p-1) / p} \int_{B} f^{+}(x, t) d x \\
& \geqslant \frac{n 2^{n+1}}{2^{n} \omega_{n} a^{n-2}} \int_{B} f^{+}(x, t) d x .
\end{aligned}
$$

Since $\omega_{n} a^{n} / n=\int_{B} d x$ is the hypervolume of $B$, the prescription of the theorem entails that

$$
k \tau \leqslant-2 a^{2} \lim _{t \rightarrow \infty} \int_{B} f^{+}(x, t) d x / \int_{B} d x .
$$

This may be compared with the prescription of Theorem 4, which, when $k$ is constant and, therefore $\gamma=(b-a) / k$, can be written as

$$
k \tau=-\frac{1}{4}(b-a)^{2} \lim _{t \rightarrow \infty} \int_{a}^{b} f^{+}(x, t) d x / \int_{a}^{b} d x .
$$

\section{REFERENCES}

[1] W. A. Day, Positive deflections of elastic beams, Quart. Appl. Math. 44, 327-336 (1986)

[2] S. Friedland, On an inequality related to certain totally positive Green's functions, Quart. Appl. Math. 44, 337-338 (1986)

[3] G. Hellwig, Partial differential equations, Blaisdell, New York, Toronto, London, 1964 